\title{
Effect of transverse perforations on fluid loading on a long, slender plate at zero incidence
}

\author{
Filippo Carlo Bossi ${ }^{1 *}$, Oleksandr Barannyk ${ }^{2}$, Mostafa Rahimpour ${ }^{2}$, Stefano Malavasi $^{1}$, Peter Oshkai $^{2}$ \\ ${ }^{1}$ Department of Civil and Environmental Engineering, Hydraulic Section, Politecnico di Milano, piazza Leonardo da Vinci 32, 20133, \\ Milano, Italy. \\ ${ }^{2}$ Department of Mechanical Engineering, Institute for Integrated Energy System, P.O. Box 1700, Stn. CSC, Victoria, BC V8W 2Y2, Canada. \\ * Corresponding author. Tel.: +39 02 2399 6260. Fax: +3902 2399 6298. E-mail: filippocarlo.bossi@polimi.it
}

\begin{abstract}
This paper reports the results of experimental investigations of flow-induced loading on perforated and solid flat plates at zero incidence with respect to the incoming flow. The plates had a streamwise length to transverse thickness ratio of 23.5. The effect of the perforations was investigated for three different perforation diameters. The results corresponding to the perforated plates were compared with the reference case of the solid plate (no perforations) at five inflow velocities. We quantified the effect of the perforations on the unsteady fluid loading on the plate in terms of the variations of the corresponding Strouhal number, the mean drag coefficient and the fluctuating lift coefficient as functions of the Reynolds number and the perforation diameter. The results indicate that the loading was dominated by the dynamics of the wake. In particular, increasing the perforation diameter resulted in a wider wake, corresponding to the increase in mean drag coefficient and the decrease in the Strouhal number. Onset of coupling between the vortex shedding and the transverse oscillations of the plate was manifested as a rapid increase in the fluctuating lift coefficient, as the perforation diameter exceeds the plate thickness.
\end{abstract}

Keywords: Perforated plate; Flow-induced loading; Flow-induced vibration.

\section{INTRODUCTION}

Flows over rectangular plates are common in a wide range of engineering systems, and they have been extensively studied over the years. A number of studies examined the dependence of the forces and the vibration frequencies on the Reynolds number (Okajima, 1982), chord-to-thickness ratio of the plate (Nakamura et al., 1991), array configurations (Guillaume and LaRue, 2001; Parker, 1966) and wall confinement (Guillaume and LaRue, 2005; Malavasi and Guadagnini, 2007; Malavasi and Zappa, 2008), among other effects. However, the effect of transverse perforations on the loading on plates of finite thickness has not been adequately addressed.

A framework for understanding the complex fluid-structure interactions (FSI) for the case of a stationary solid plate at zero incidence in terms of the Strouhal number, $S t=f L / U$, where $f$ is the characteristic frequency of the flow and/or structure oscillations, $L$ is the characteristic length, and $U$ is the characteristic flow velocity, is provided by Parker and Welsh (1983), Nakamura and Nakashima (1986), Nakamura et al. (1991, 1996) and Naudascher and Rockwell (1994). Considering experimental results with a Reynolds number based on the plate thickness, $R e_{t}=U t / v$ (where $v$ is the kinematic viscosity of the fluid) of $1 \cdot 10^{3}<R e_{t}<3.5 \cdot 10^{4}$, a change of the chord-tothickness ratio (side ratio) $c / t$ has a significant effect on the flow-induced vibrations. For a plate with sharp edges and a side ratio of $0.1<c / t<30$, it was found that the Strouhal number based on thickness $\left(S t_{t}=f t / U\right)$ varied roughly from 0.05 to 0.2 with a particular stepping behavior that corresponds to different vortex shedding mechanisms. Particularly related to the present study, which considers sharp edged plates with $c / t=23.5$, is the trailing edge vortex shedding mechanism. In this regime, the shear layers that form at the leading edge of the plate reattach upstream of the trailing edge, and the vortex street formed in the wake of the plate is not directly related to the separation bubbles that exist at the leading edge.
For bluff plates, the Strouhal number and the drag strongly depend on the dynamics of the wake, particularly the region of the vortex formation (Roshko, 1954, 1955). Specifically, the Strouhal number is inversely proportional to the width of the wake $d$ ', while the pressure drag is proportional to $d$ '. The wake Strouhal number $S t_{d^{\prime}}=f d^{\prime} / U_{s}$, where $U_{s}$ is the velocity on the free streamline at the point of shear layer separation, generally does not depend on the bluff body that generated the wake. However, when the thickness of the separated shear layers is significantly affected by the geometry of the body, such as in the present study, which considered plates with transverse perforations of different size, the vortex dynamics in the wake also depend on the formation of a boundary layer over the body surfaces. Therefore the Strouhal number and the pressure drag coefficient corresponding to different bodies are expected to be different, even at the same wake Reynolds number $R e_{d^{\prime}}=$ $U_{s} d^{\prime} / v$, where $v$ is the kinematic viscosity of the fluid (Roshko, 1955).

Although the Strouhal number trends of the wake dynamics are well-established for solid plates and, to a lesser extent, for perforated plates, the trend of the drag coefficient, defined as $C_{D}=2 D /\left(\rho U^{2} S\right)$, where $D$ is the drag force, $\rho$ is the fluid density, $U$ is the characteristic flow velocity and $S$ is the characteristic surface area, as a function of the Reynolds number and the plate geometry is not well-established. For a bluff solid plate of comparable side ratio to the current case, no information about the drag coefficient has been reported in literature to the best of the authors' knowledge. For the case of perforated plates, a qualitative comparison with the current results can be carried out by considering the numerical study of Bao et al. (2012), who numerically investigated an inline array of six square cylinders with adjustable spacing subject to an incompressible flow at $R e=100$ (based on the cylinder side length). Analyzing the effect of the cylinder spacing on the Strouhal number and the drag coefficient, Bao et al. (2012) identified different flow patterns that depend on the spacing between the cylinders. 
Small gaps were characterized by single flow recirculation cells that form between the cylinders. As the spacing between the cylinders increased, symmetric and antisymmetric patterns of vortex shedding were observed. The present study considers the overall effect that the flow oscillations over the individual perforations have on the boundary layers that form over the plate and, in turn on the wake dimensions and the resulting force coefficients.

Turbulent flow over a perforated plate can generate substantial levels of radiated acoustic noise and vibrations (Howe, 1990, 1997a; Celik and Rockwell, 2004) where sources corresponding to the individual perforations can be modeled as either monopoles (Ffowcs Williams, 1972) or linearly distributed vortex sheets, depending on the relative dimensions of the perforations relative to the displacement thickness of the boundary layer (Howe, 1997a). Coupling of the flow oscillations with acoustic pressure pulsations has received significant attention (Howe, 1986, 1997b; Malavasi and Bossi, 2012; Oshkai and Velikorodny, 2013; Parker, 1966; Parker and Welsh, 1983; Welsh et al., 1984). Resonance in many common configurations of engineering significance, such as arrays of parallel plates or splitter plates in pipelines, is often dominated by acoustic effects, rather than vibration of the plates (Oshkai and Velikorodny, 2013; Parker, 1966). In contrast to the previous investigations that focused on the flow-acoustic coupling, the present study considers the case where the frequency of vortex shedding was well separated from the frequencies of the available acoustic modes. Therefore, acoustic resonance did not play a significant role, and the unsteady loading and vibrations were caused entirely by hydrodynamic effects. In the present investigation, the amplitude of the plate oscillations was negligible, with the exception of a single case, where onset of a lockon between the structural vibrations and the vortex shedding was observed.

\section{EXPERIMENTAL SYSTEM AND TECHNIQUES Flow facility}

The experiments were conducted in a free-surface water channel located in the Fluid Mechanics Laboratory at the University of Victoria. The flow was conditioned using five fine mesh screens and a polycarbonate honeycomb section with round cells with the diameter of $4 \mathrm{~mm}$. A converging section with a contraction area ratio of $6: 1$ was located upstream of the test section. The test section had a square cross-section of
$45 \mathrm{~cm} \mathrm{x} 45 \mathrm{~cm}$ and a length of $250 \mathrm{~cm}$.

The plates, which were positioned parallel to the flow, were cantilevered at the top edge and attached to the frame of the water channel by a support structure as shown in Fig. 1(a). A load cell that enabled measurements of the forces acting on the plate was attached to the end of the plate and the supporting frame. Gaps of $2.5 \mathrm{~mm}$ were left above and below the plate to avoid contact between the plate and the walls of the test section due to flow-induced motion. For all the cases considered herein, the water depth $W$ was equal to $41.5 \mathrm{~cm}$, so that the freesurface level coincided with the edge of the clamp, as shown in Fig. 1(a).

\section{Perforated plates}

We studied three flat plates with staggered distribution of circular perforations shown in Fig. 1(b). The plates were positioned at the incidence angle of $0^{\circ}$ with respect to the oncoming flow. For all three plates, the distance between perforations was equal to the perforation diameter, as a consequence, in our setup, the plate porosity is univocally defined by one parameter, the perforation diameter $\left(d_{p}\right)$. The plates were designed to have a comparable porosity, with the equivalent area ratio $\beta=$ $\left(n A_{p} / A_{t}\right)^{0.5}=0.4$, where $n$ is the number of perforations, $A_{p}$ is the area of a single perforation, $A_{t}$ is the total wetted area of the plate. $\beta$ was defined in accordance with Idelchik (1986) and taking in account the end-plate effects. The investigation also included a reference case of a solid plate (without perforations). Plates with perforations of different diameters were studied at several inflow velocities.

The geometrical characteristics of the four plates under consideration (the ratio of the perforation diameter to the plate thickness $d_{p} / t$ and the number of perforations $n$ ) are listed in Table 1. The plates had length $c=292.1 \mathrm{~mm}$ in the streamwise direction, width $w=412.8 \mathrm{~mm}$ and thickness $t=12.4 \mathrm{~mm}$, which corresponded to the side ratio $c / t=23.5$. Particular attention was given to defining the distance between perforations and between the perforations and the edges of the plates so that the ratios of these parameters to the perforation diameter $d_{p}$ remained constant (equal to unity) between the three considered perforation patterns. Thus, the parameter space was limited to the perforation diameter $d_{p}$ and the inflow velocity $U$. The solid plate and the perforated plates had natural vibrational frequencies of $f_{0}=5.1 \mathrm{~Hz}$ and $f_{0}=7.8 \mathrm{~Hz}$, respectively. The frequencies were determined by free decay tests in quiescent water.

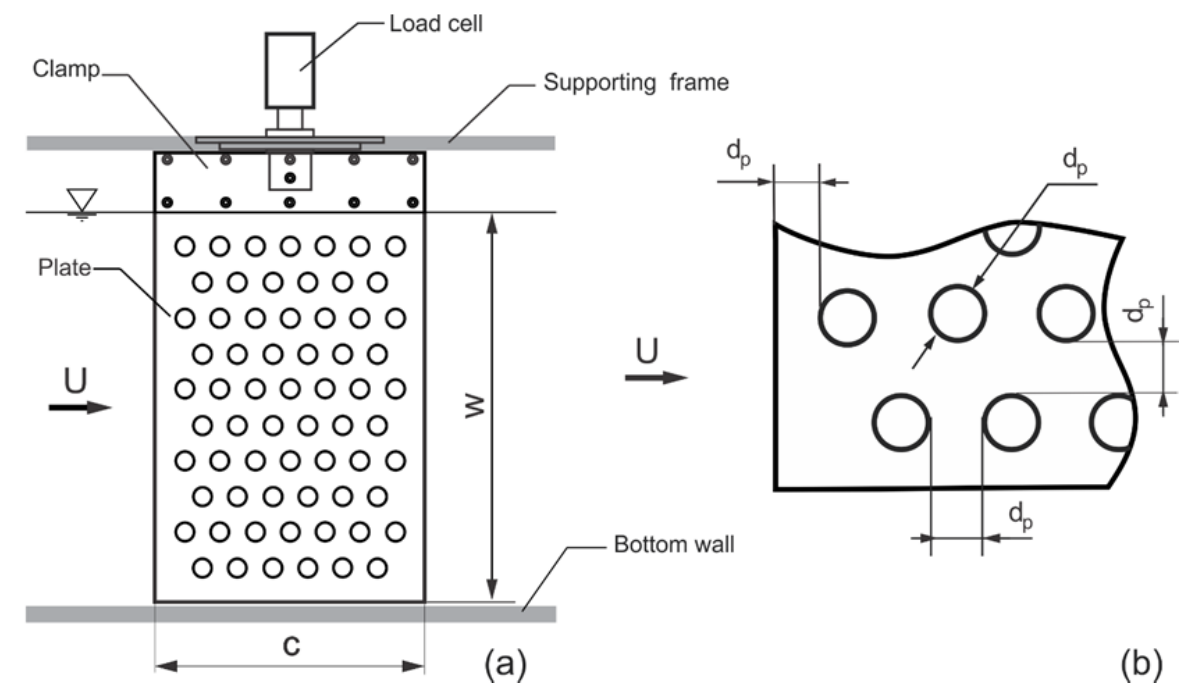

Fig. 1. (a) Schematic of the experimental system. (b) Close-up of the perforation pattern. 
Table 1. Characteristic dimensions of the plates.

\begin{tabular}{lcc}
\hline Perforation pattern & $d_{p} / t$ & $n$ \\
\hline Solid plate & - & - \\
P1 & 0.76 & 304 \\
P2 & 1.02 & 168 \\
P3 & 1.59 & 65 \\
\hline
\end{tabular}

\section{Inflow conditions}

A General Oceanics 2030R6 flow meter with a highresolution rotor for low-speed application was used to record the flow speed upstream of the test section. Inside the test section, the presence of the plate and its supporting structure resulted in the blockage ratio of $12.7 \%$. The Reynolds number based on the thickness of the plate $R e_{t}=U t / v$ varied in the range $2.7 \cdot 10^{3} \leq R e_{t} \leq 7.1 \cdot 10^{3}$. Increasing the $\mathrm{Re}_{\mathrm{t}}$ beyond this range resulted in non-negligible wave formation at the free surface. The turbulence intensity upstream of the plate was equal to $2.5 \%$. The water temperature was kept around $22^{\circ} \mathrm{C}$.

\section{Unsteady force measurements}

The unsteady flow-induced loading on the plate was measured by a tri-axial load cell (Novatech F233). The load cell was rigidly connected to the supporting structure and could measure forces $\left(F_{x}\right.$ and $\left.F_{y}\right)$ and torque $(M)$ along three mutually perpendicular axes. Each output channel of the load cell was connected to a dedicated amplifier (Novatech SGA/A). Low-pass filtering was applied at the time of data acquisition to avoid aliasing and to reduce noise in the sampled signal.

The calibration center of the load cell corresponded to the center of gravity of the plate, considering only its wetted portion. The uncertainty of the force measurements was estimated to be $\pm 0.7 \%$ of the rated value for all the three axes. Zero levels of the signals in quiescent water were determined at the beginning of each experiment. To optimize spectral resolution, the data were recorded at a sampling frequency of $200 \mathrm{~Hz}$ for ten minutes. To analyze the data in frequency domain, Fourier transform was applied to the signals, multiplied by ten nonoverlapped Hamming windows to reduce leakage error.

\section{EFFECT OF PERFORATION DIAMETER AND INFLOW VELOCITY ON FLOW-INDUCED FORCES Frequency domain analysis}

The frequency spectra of the flow-induced forces were calculated based on the signal of the torque channel of the load cell, which showed the highest signal-to-noise ratio of the three axes. The average value was removed from the data before conversion to the frequency domain. The frequency resolution was equal to $1.5 \cdot 10^{-3} \mathrm{~Hz}$, and the calculated spectra covered the frequency range of up to $100 \mathrm{~Hz}$. The plots presented herein show a portion of this range, containing the frequencies of interest.

The waterfall plot of Fig. 2 shows variation of the power spectral density (PSD) of the flow-induced loading as a function of the frequency and the inflow velocity for the reference case of the solid plate (without perforations). The predominant spectral peaks corresponded to the vortex shedding frequencies in the wake of the plate. These frequencies varied linearly with the inflow velocity, as indicated in Fig. 2 by the dashed line, and were used to calculate the Strouhal numbers of the flowinduced loading. The magnitudes of the predominant peaks are indicated by the labels in Fig. 2 . At $U=0.29 \mathrm{~m} / \mathrm{s}$, the vortex shedding frequency coincided with the natural vibrational frequency $f_{0}=5.1 \mathrm{~Hz}$, which resulted in an increase in the peak value of the PSD.

The trends of the PSD corresponding to the perforation patterns P1 and P2, shown in Figs. 3 and 4, respectively, were qualitatively similar to those corresponding to the solid plate. The spectral peaks corresponding to the natural vibrational frequency $f_{0}=7.8 \mathrm{~Hz}$ were more pronounced, but the peaks corresponding to the vortex shedding still followed a linear trend, as indicated by the dashed lines.

In the case of the perforation pattern $\mathrm{P} 1$, with the smallest considered diameter of the perforations $d_{p} / t=0.76$, the spectral peak corresponding to the natural vibrational frequency $f_{0}=7.8$ $\mathrm{Hz}$ was not detected at the lowest considered value of the inflow velocity $U=0.2 \mathrm{~m} / \mathrm{s}$. As the inflow velocity increased to $U=0.29 \mathrm{~m} / \mathrm{s}$, the peak became pronounced, reaching the corresponding value of the PSD of $2.89 \cdot 10^{-7}(\mathrm{Nm})^{2} / \mathrm{Hz}$. At the higher values of the inflow velocity, the PSD of the peak corresponding to $f_{0}=7.8 \mathrm{~Hz}$ decreased again, which indicates that the energy of the incoming flow was directed into the flow oscillations in the wake, rather than the vibrations of the plate.

As the diameter of the perforations increased to $d_{p} / t=1.02$ in the case of the perforation pattern P2, shown in Fig. 4, the onset of lock-on between vortex shedding and structural vibrations was observed, as the spectral peak corresponding to the natural vibrational frequency $f_{0}=7.8 \mathrm{~Hz}$ became and remained dominant, with the corresponding PSD value exceeding that of the peak corresponding to the vortex shedding, as the inflow velocity reached and exceeded $U=0.37 \mathrm{~m} / \mathrm{s}$. At $U=0.46 \mathrm{~m} / \mathrm{s}$, the peak corresponding to the vortex shedding was still detectable, while the peak associated with the natural vibrational frequency increased to $2.24 \cdot 10^{-5}(\mathrm{Nm})^{2} / \mathrm{Hz}$, indicating that the energy of the incoming flow was largely directed to generating structural vibrations. The frequency coincidence between the structural vibrations and the vortex shedding was observed at $U=0.55 \mathrm{~m} / \mathrm{s}$, which resulted in a substantial increase in the PSD value of the corresponding spectral peak.

In the case of the perforation pattern P3, which had the largest considered diameter of the perforations $d_{p} / t=1.59$, shown in Fig. 5, the frequency coincidence between the vortex shedding and the vibrations of the plate was observed at $U=0.46 \mathrm{~m} / \mathrm{s}$. As the inflow velocity increased to $U=0.55 \mathrm{~m} / \mathrm{s}$, the predominant spectral peak with the corresponding PSD value of $4.66 \cdot 10^{-3}$ $(\mathrm{Nm})^{2} / \mathrm{Hz}$ remained locked-on to the natural vibrational frequency $f_{0}=7.8 \mathrm{~Hz}$. A weak peak at the vortex shedding (Strouhal) frequency was also observed at this value of the inflow velocity. At this condition, the highest amplitude of vibration of the plate, across all considered cases, was observed $\left(A_{v}=1 \mathrm{~mm}\right)$, as measured by means of high-speed video recording with a framing rate exceeding the predominant vibrational frequency by two orders of magnitude.

Onset of the lock-on between the vortex shedding and the structural vibrations occurred at the lower inflow velocities, as the perforation diameter increased, which can be attributed to reduced stiffness of the plates with larger perforations.

\section{Effect of the perforation diameter on the vortex shedding frequency}

The data shown in the spectral form in Figs. 2-5 were used to calculate dimensionless frequencies $f / f_{0}$ corresponding to the vortex shedding and the structural vibrations, which are shown in Fig. 6 as functions of reduced velocity $U^{*}=U /\left(f_{0} t\right)$. 


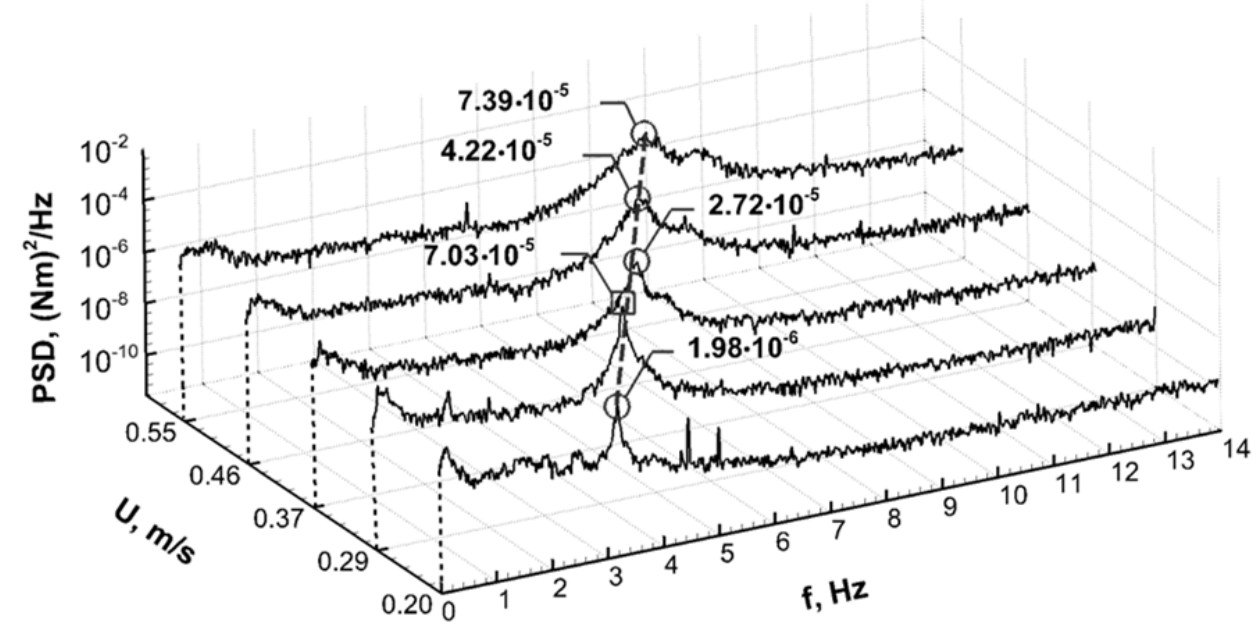

Fig. 2. Power spectral density of the flow-induced loading on the solid plate as a function of the inflow velocity and frequency. Spectral peaks: $\circ$ vortex shedding frequencies, $\square$ structural vibrations.

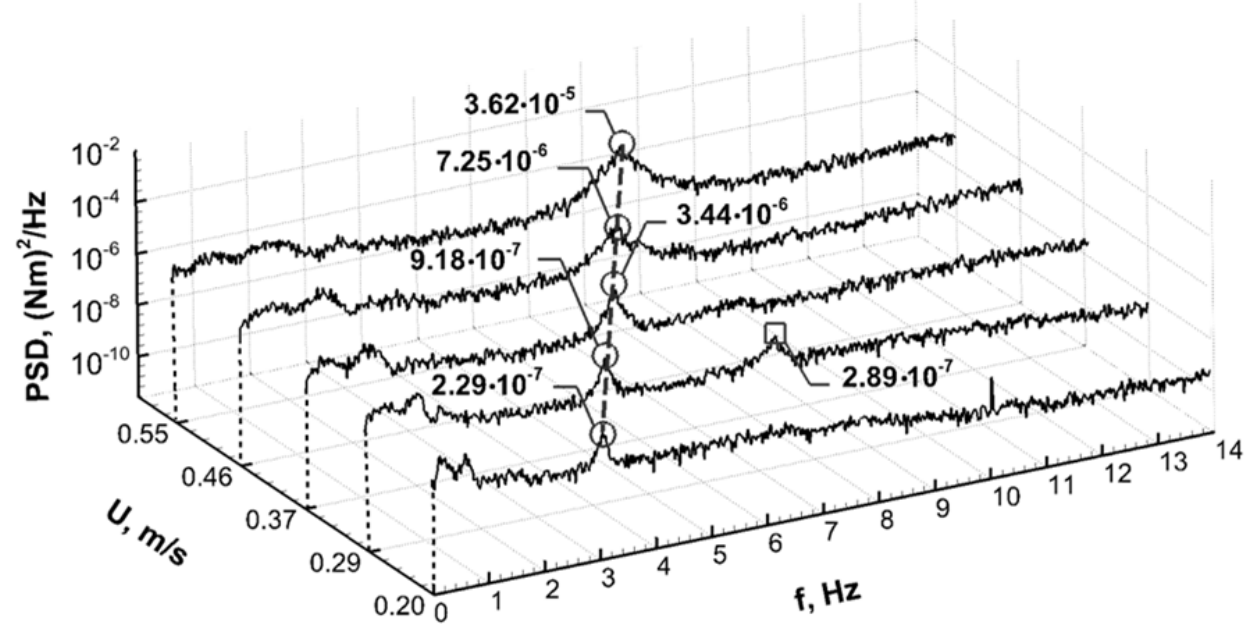

Fig. 3. Power spectral density of the flow-induced loading on the plate with a perforation pattern $\mathrm{P} 1\left(d_{p} / t=0.76\right)$ as a function of the inflow velocity and frequency. Spectral peaks: $\circ$ vortex shedding frequencies, $\square$ structural vibrations.

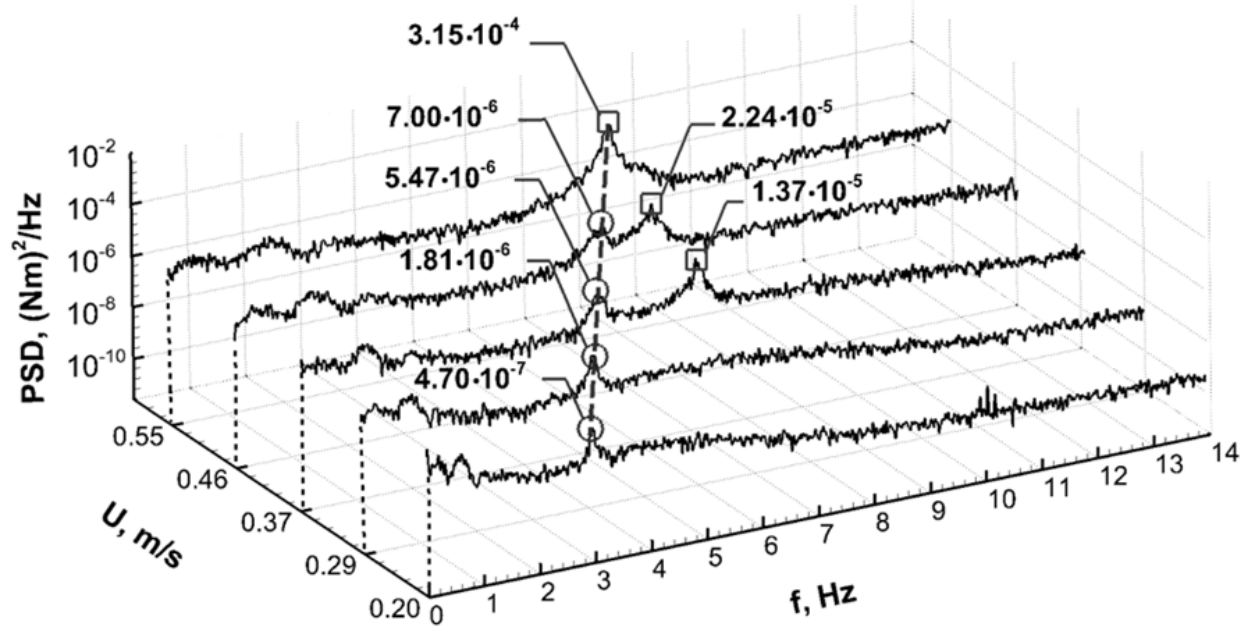

Fig. 4. Power spectral density of the flow-induced loading on the plate with a perforation pattern $\mathrm{P} 2\left(d_{p} / t=1.02\right)$ as a function of the inflow velocity and frequency. Spectral peaks: $\circ$ vortex shedding frequencies, $\square$ structural vibrations. 


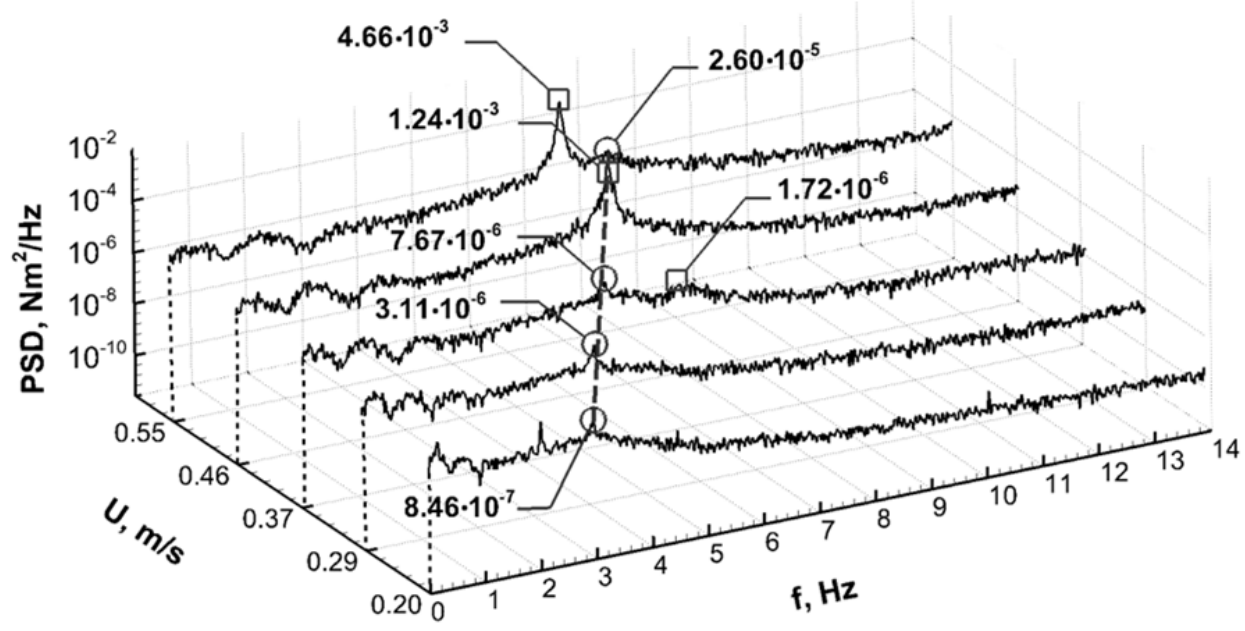

Fig. 5. Power spectral density of the flow-induced loading on the plate with a perforation pattern P3 $\left(d_{p} / t=1.59\right)$ as a function of the inflow velocity and frequency. Spectral peaks: $\circ$ vortex shedding frequencies, $\square$ structural vibrations.

The open and solid symbols indicate the frequencies of the structural vibrations and the vortex shedding, respectively.

The dash-dot line represents the natural vibrational frequency in quiescent water $f_{0}$. For all cases where the plate vibrations were detected, the corresponding frequencies were within $20 \%$ of $f_{0}$.

The dimensionless frequencies of the vortex shedding varied linearly with the reduced velocity, but the trends had different proportionality coefficients, corresponding to the Strouhal numbers $S t_{t}=f t / U$. The dashed lines indicate the upper and the lower bounds of the observed Strouhal numbers $\left(0.192<S t_{t}<\right.$ $0.177)$.

The plot of Fig. 6 shows small but consistent decrease of approximately $10 \%$ for $S t_{t}$ with the increase of the perforation diameter $d_{p} / t$. This trend is also evident in the plot of Fig. 7, which shows variation of the Strouhal number $S t_{t}$ as a function of the Reynolds number $R e_{t}=U t / v$. This result indicates that increasing the perforation diameter results in formation of thicker boundary layers at the trailing edge of the plate and, in turn, larger wake width $d^{\prime}$ and lower frequency of vortex formation $f$, which is consistent with the results of Roshko (1954, 1955) for a generalized bluff body.

Moreover, a trend that is qualitatively similar to the decrease in the Strouhal number with the increase of the perforation diameter was observed in the case of a row of square cylinders that was numerically simulated by Bao et al. (2012). The Strouhal numbers decreased when the separation between the cylinders was increased. The differences in the configurations and the range of the Reynolds numbers between the current study and that of Bao et al. (2012) did not allow quantitative comparison of the results, but the general trend suggests that flow separation over the individual perforations, similar to the gaps in the row of cylinders, resulted in the increase in the length of the vortex formation in the wake, corresponding to the decrease in the vortex shedding frequency represented by the Strouhal number.

For all considered plates, the Strouhal number was weakly dependent on the Reynolds number. In particular, an increase of $R e_{t}$ by approximately $270 \%$ resulted in a decrease of $S t_{t}$ of $4 \%$, on average, as shown in Fig. 7. This trend is in agreement with the results reported by Rockwell and Naudascher (1994). Moreover, the range of the observed Strouhal numbers is consistent with the results of Parker and Welsh (1983) and Knisely (1990)

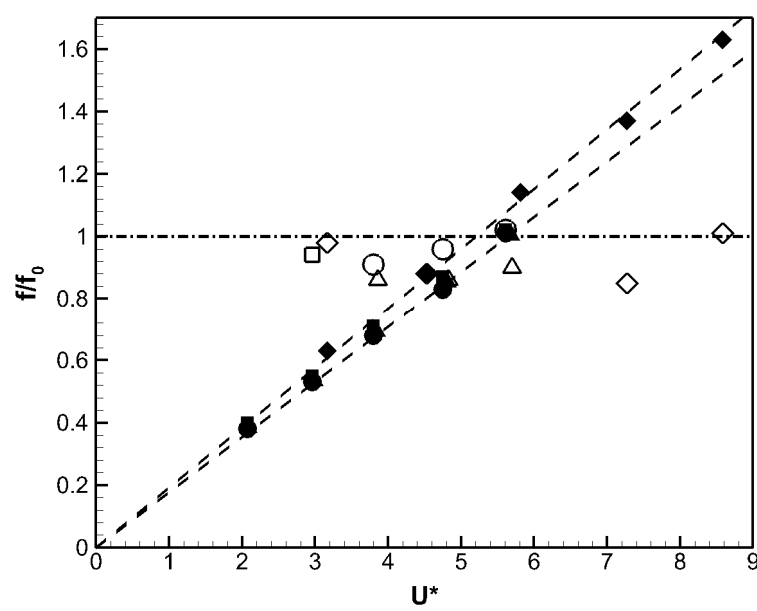

Fig. 6. Dimensionless frequencies of the vortex shedding (solid symbols) and the plate vibrations (open symbols) as functions of the reduced velocity. Perforation patterns: $\diamond$ solid plate, $\square$ P1, $\circ$ P2 and $\Delta \mathrm{P} 3$.

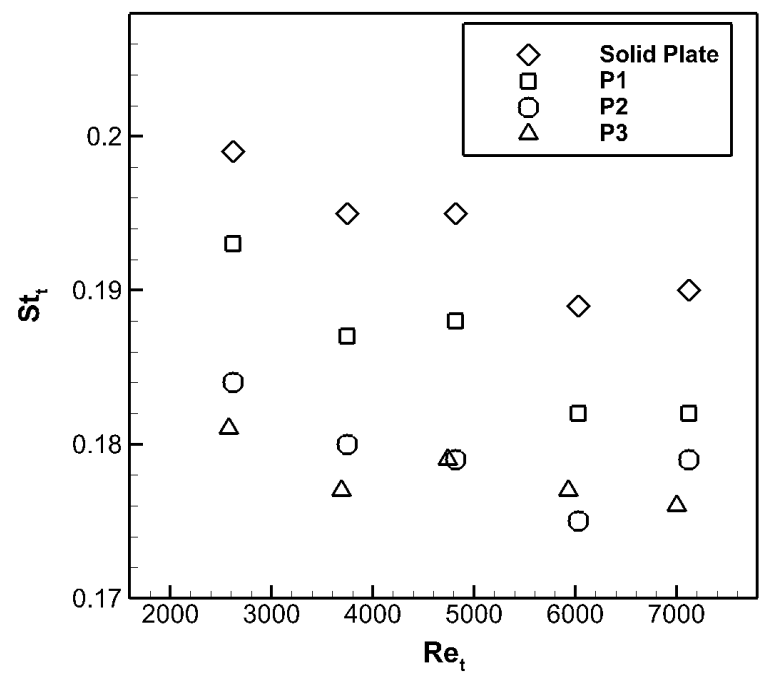

Fig. 7. Variation of the Strouhal number as a function of the Reynolds number. Perforation patterns: $\diamond$ solid plate, $\square \mathrm{P} 1, \circ \mathrm{P} 2, \Delta \mathrm{P} 3$. 


\section{Effect of the perforation diameter on the magnitude of flow-induced forces}

Increasing the diameter of the perforations $d_{p} / t$ had a pronounced effect on the magnitude of the drag (streamwise) force acting on the plate, represented by the mean drag coefficient, defined as follows:

$\overline{C_{D}}=\frac{\bar{D}}{\frac{1}{2} \rho U^{2} w t}$,

where $\bar{D}$ is the mean drag force, and the product of the chord length and the thickness of the plate $w t$ represented the characteristic surface area of the plate, perpendicular to the inflow direction.

The evolution of the mean drag coefficient as a function of the Reynolds number for cases of different perforation patterns is shown in Fig. 8. For all perforation patterns, the drag coefficient showed a slight initial decrease as the Reynolds number reached the value of $R e_{t}=3800$, followed by a subsequent increase at $R e_{t}=4800$. Overall, the drag coefficient did not change significantly over the considered range of the Reynolds numbers, but it varied substantially with the perforation diameter. Specifically, as the perforation pattern changed, which corresponded to an increase in the dimensionless perforation diameter $d_{p} / t$, the drag coefficient monotonically increased by approximately $14 \%$. It has been established that the drag coefficient of a bluff body increases as the wake width $d$ ' increases (Roshko, 1954, 1955). Thus, the results shown in Fig. 8 support the conclusion drawn based on the analysis of dimensionless vortex shedding frequencies shown in Fig. 7 that the shear layers that formed on the plate with the larger perforations were thicker than those corresponding to the smaller perforations. This effect is likely due to the higher amplitude of transverse flow oscillations that were established across the openings of the larger perforations, as reported by Bao et al. (2012) and Howe (1997c), among others. Quantitative confirmation of this hypothesis requires flow visualization in the vicinity of the individual perforations, which is the subject of a future investigation.

The magnitude of the fluctuating lift coefficient, plotted in Fig. 9 as a function of the Reynolds number, was related to structural vibrations of the plate that are discussed in terms of their frequencies in subsection "Frequency domain analysis". The fluctuating lift coefficient was defined as follows:

$$
C_{L}^{\prime}=\frac{L^{\prime}}{\frac{1}{2} \rho U^{2} w t} .
$$

Here, $L^{\prime}$ is the root-mean-square (rms) value of the lift force $L$, which was calculated according to Eq. (3):

$$
L^{\prime}=\sqrt{\frac{1}{T} \int_{0}^{T}(L-\bar{L})^{2} d \tau},
$$

where $\bar{L}$ is the mean lift force, $\tau$ is time and $T$ is the duration of the data acquisition (ten minutes).

At low Reynolds numbers, the fluctuating lift coefficient of the solid plate was substantially higher than that of the perforated plates. The $C_{L}^{\prime}$ reached an asymptotic value in the range

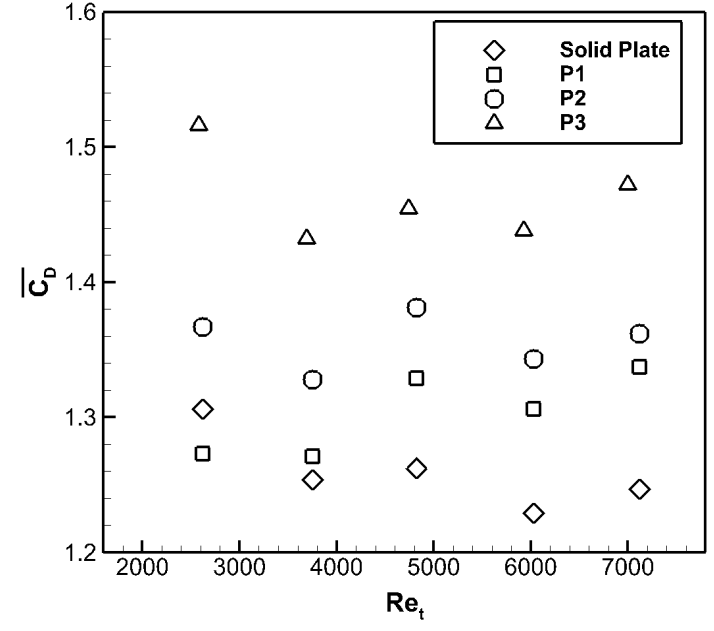

Fig. 8. Variation of the mean drag coefficient as a function of the Reynolds number. Perforation patterns: $\diamond$ solid plate, $\square \mathrm{P} 1, \circ \mathrm{P} 2, \Delta \mathrm{P} 3$.

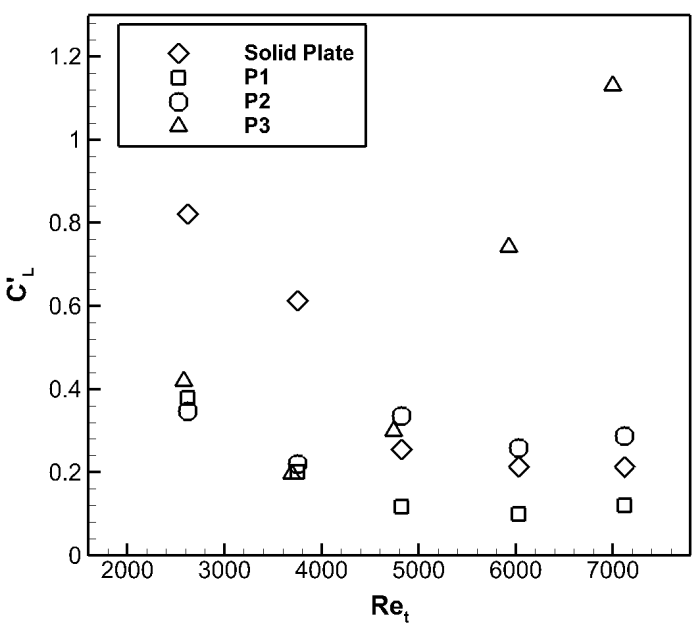

Fig. 9. Variation of the fluctuating lift coefficient as a function of the Reynolds number. Perforation patterns: $\diamond$ solid plate, $\square$ P1, $\circ \mathrm{P} 2, \Delta \mathrm{P} 3$.

$0.10<C_{L}{ }^{\prime}<0.29$ for the cases of the solid plate and the plates with perforation patterns $\mathrm{P} 1$ and $\mathrm{P} 2$ as the Reynolds number increased above $R e_{t}=4800$. In contrast to these plates, the plate with the perforation pattern $\mathrm{P} 3$, which had the largest diameter of the perforations $d_{p} / t$, exhibited a different trend - the corresponding fluctuating lift coefficient monotonically increased as the Reynolds number increased above $R e_{t}=3800$. This result can be attributed to two factors. First, the plate with the perforation pattern P3 had the lowest stiffness and exhibited the largest amplitude of the structural vibrations, corresponding to the onset of lock-on between the vibrations and the vortex shedding, which is described in subsection "Frequency domain analysis". Second, the large perforations likely resulted in vortex shedding at the individual perforations and the associated fluctuations of the transverse velocity component in the vicinity of the perforations, by analogy with the row of square cylinders studied by Bao et al. (2012), who showed that the vortex shedding was consistently observed in the region between the cylinders, corresponding to an increase in the fluctuating lift coefficient, as the separation between the cylinders exceeded the side length of the cylinder, which corresponds (as a 2D approximation) to the perforation diameter equal to the plate thickness in the current study. 


\section{CONCLUSIONS}

The effect of transverse perforations on the unsteady loading on bluff plates at zero incidence was studied experimentally. The Strouhal number corresponding to the vortex shedding from the trailing edge of the plate and the mean drag coefficient were found to be strongly dependent on the perforation diameter, while the Reynolds number, within the considered range of $2.7 \cdot 10^{3} \leq R e_{t} \leq 7.1 \cdot 10^{3}$, had minor influence.

In particular, the Strouhal number decreased with the increase of the perforation diameter, while the mean drag coefficient increased. For the reference case of the solid plate (without perforations), the trend of the Strouhal number was consistent with the results available in the literature. For the cases of the perforated plates, a qualitative similarity was observed between the current data and that corresponding to an array of square cylinders, where the frequency of the predominant spectral peaks decreased with the increase of the spacing between the cylinders. Moreover, the increase of the mean drag coefficient for the case of the perforated plates was similar to the corresponding trend in the case of the square cylinders, where increasing the separation between the cylinders allowed development of unsteady flow structures between them.

Increasing the diameter of the perforations resulted in formation of thicker shear layers at the trailing edge of the plate, corresponding to a wider wake and therefore, a higher mean drag coefficient and lower Strouhal number, compared to the cases of smaller perforations and the reference case of the solid plate.

As the perforation diameter exceeded the plate thickness, the onset of coupling between transverse vibrations of the plate and the vortex shedding was observed, which was accompanied by a significant increase in the fluctuating lift coefficient.

Acknowledgements. The authors gratefully acknowledge the financial contribution of PIBIVIESSE s.r.l. to the Ph.D. scholarship of Filippo Bossi and financial support of the Natural Sciences and Engineering Research Council of Canada (NSERC) under a Discovery Grant.

\section{REFERENCES}

Bao, Y., Wu, Q., Zhou, D., 2012. Numerical investigation of flow around an inline square cylinder array with different spacing ratios. Comput. Fluids, 55, 118-131. DOI: 10.1016/j.compfluid.2011.11.011.

Celik, E., Rockwell, D., 2004. Coupled oscillations of flow along a perforated plate. Phys. Fluids, 16, 1714-1724, DOI: 10.1063/1.1661625.

Ffowcs Williams, J.E., 1972. The acoustics of turbulence near sound-absorbent liners. J. Fluid Mech., 51, 737-749, DOI: $10.1017 /$ S0022112072001338.

Guillaume, D.W., LaRue, J.C., 2001. Comparison of the vortex shedding behavior of a single plate and a plate array. Exp. Fluids, 30, 22-26, DOI: 10.1007/s003480000130.

Guillaume, D.W., LaRue, J.C., 2005. Comparing the flow on the bounded and unbounded sides of a plate. Int. J. Heat Mass Tran., 48, 1384-1387. DOI: 10.1016/j.ijheatmasstransfer.2004.10.012.

Howe, M.S., 1986. Attenuation of sound due to vortex shedding from a splitter plate in a mean flow duct. J. Sound Vibr., 105, 385-396. DOI: 10.1016/0022-460X(86)90167-7.

Howe, M.S., 1990. Sound produced by turbulent flow over a perforated inlet. J. Sound Vibr. 139, 227-240. DOI: 10.1016/0022-460x(90)90884-3.
Howe, M.S., 1997a. Sound generated by turbulence and discrete vortices interacting with a perforated elastic plate in low-Machnumber flow. Q. J. Mech. App. Math., 50, 279-301. DOI: 10.1093/qjmam/50.2.279.

Howe, M.S., 1997b. Influence of cross-sectional shape on the conductivity of a wall aperture in mean flow. J. Sound Vibr., 207, 601-616. DOI: 10.1006/jsvi.1997.1103.

Howe, M.S., 1997c. Edge, cavity and aperture tones at very low Mach numbers. J. Fluid Mech., 330, 61-84. DOI: 10.1017/s0022112096003606.

Idelchik, I.E., 1986. Handbook of Hydraulic Resistance. $2^{\text {nd }}$ ed. Hemisphere Publishing Corporation, Washington, DC.

Knisely, C.W., 1990. Strouhal numbers of rectangular cylinders at incidence: A review and new data. J. Fluids Struct., 4, 371-393. DOI: 10.1016/0889-9746(90)90137-t.

Malavasi, S., Bossi, F.C., 2012. Experimental analysis of aerodynamic noise generation in a rotary control valve. In: Meskell, C., Bennett, G. (Eds.): Proc. 10th International Conference on Flow-Induced Vibrations \& Flow-Induced Noise (FIV2012). Dublin, Ireland, pp. 697-703.

Malavasi, S., Guadagnini, A., 2007. Interactions between a rectangular cylinder and a free-surface flow. J. Fluid Struct., 23, 1137-1148, 10.1016/j.jfluidstructs.2007.04.002.

Malavasi, S., Zappa, E., 2008. Fluid-dynamic forces and wake frequencies on a tilted rectangular cylinder near a solid wall. Meccanica, 44, 91-101. DOI: 10.1007/s11012-008-9154-2.

Nakamura, Y., Nakashima, M., 1986. Vortex excitation of prisms with elongated rectangular, $\mathrm{H}$ and [vertical, dash] crosssections. J. Fluid Mech., 163, 149-169. DOI: 10.1017/S0022112086002252.

Nakamura, Y., Ohya, Y., Tsuruta, H., 1991. Experiments on vortex shedding from flat plates with square leading and trailing edges. J. Fluid Mech., 222, 437-447. DOI: 10.1017/s0022112091001167.

Nakamura, Y., Ohya, Y., Ozono, S., Nakayama, R., 1996. Experimental and numerical analysis of vortex shedding from elongated rectangular cylinders at low Reynolds numbers 200103. J. Wind Eng. Ind. Aerod., 65, 301-308. DOI: 10.1016/s0167-6105(97)00048-2.

Naudascher, E., Rockwell, D., 1994. Flow-Induced Vibrations: an Engineering Guide. $2^{\text {nd }}$ ed. A.A. Balkema Publishers, Rotterdam, the Netherlands.

Okajima, A., 1982. Strouhal numbers of rectangular cylinders. J. Fluid Mech., 123, 379-398. DOI: 10.1017/s0022112082003115.

Oshkai, P., Velikorodny, A., 2013. Flow-acoustic coupling in coaxial side branch resonators with rectangular splitter plates. J. Fluid Struct., $\quad 38, \quad 22-39$. DOI: 10.1016/j.jfluidstructs.2012.12.008.

Parker, R., 1966. Resonance effects in wake shedding from parallel plates: Some experimental observations. J. Sound Vibr., 4, 6272. DOI: 10.1016/0022-460x(66)90154-4.

Parker, R., Welsh, M.C., 1983. Effects of sound on flow separation from blunt flat plates. Int. J. Heat Fluid Flow, 4, 113-127. DOI: 10.1016/0142-727x(83)90014-0.

Roshko, A., 1954. On the drag and shedding frequency of twodimensional bluff bodies. In: Aeronautics NACf (Eds.). Washington.

Roshko, A., 1955. On the wake and drag of bluff bodies. J. Aeronaut. Sci., 22, 124-132. DOI: 10.2514/8.3286.

Welsh, M.C., Stokes, A.N., Parker, R., 1984. Flow-resonant sound interaction in a duct containing a plate. 1. Semi-circular leading edge. J. Sound Vibr., 95, 305-323. DOI: 10.1016/0022460x(84)90670-9.
Received 31 May 2016

Accepted 1 November 2016 